\title{
Clinical significance of CD44 expression in children with hepatoblastoma
}

\author{
H.-Y. Cai ${ }^{1 *}$, B. Yu ${ }^{1 *}$, Z.-C. Feng ${ }^{2}$, X. Qi ${ }^{1}$ and X.-J. Wei ${ }^{1}$ \\ 1'Department of General Surgery, General Hospital of Beijing Military Region, \\ Beijing, China \\ ${ }^{2} \mathrm{Ba}$ Yi Children's Hospital Affiliated Beijing Military General Hospital, \\ No. 5 Nan Men Cang, East District, Beijing, China \\ *These authors contributed equally to this study. \\ Corresponding author: Z.-C. Feng \\ E-mail: fengzhichundr@163.com \\ Genet. Mol. Res. 14 (4): 13203-13207 (2015) \\ Received April 22, 2015 \\ Accepted August 28, 2015 \\ Published October 26, 2015 \\ DOI http://dx.doi.org/10.4238/2015.October.26.16
}

\begin{abstract}
The aim of this study was to investigate the expression of CD44 and its clinical significance in children suffering from hepatoblastoma (HB). CD44 expression was detected with immunohistochemistry staining in 30 samples from hepatoblastoma children and 10 normal liver tissue samples from normal children. The data obtained was statistically analyzed using the chi-square test, using the SPSS (v.11.0) software. The rate of CD44 expression was significantly higher $(66.7 \%)$ in hepatoblastoma tissues than in normal liver tissues $\left(X^{2}=4.848, P<0.05\right)$. The rate of CD44 expression was significantly higher in children with stage III or IV hepatoblastoma (83.3\%) than that in children with stage I and II hepatoblastoma $\left(X^{2}=5.625, P<0.05\right)(41.7 \%)$. Therefore, CD44 expression might play an important role in the pathogenesis, progression, and prognosis of $\mathrm{HB}$ in children.
\end{abstract}

Key words: Hepatoblastoma; Antigens; CD44; Children 


\section{INTRODUCTION}

Hepatoblastoma (HB), a primary embryonal tumor, is one of the most common among pediatric malignant solid tumors affecting the liver. It originates from undifferentiated cells of embryonic tissues, which can differentiate into the liver and bile duct epithelium cellular pluripotent stem cells (Helmberger et al., 1999). CD44 initially serves as a cell surface adhesion molecule, and subsequently mediates interactions among cells, and between the cells and the extracellular matrix. The results of previous analyses have shown the involvement of CD44 molecules in signal transduction between cells, and the regulation of tumor growth, invasion, and metastasis (Louderbough et al., 2001; Jang et al., 2001). CD44 has been recently discovered to be an important surface marker molecule in a variety of cancer stem cells, playing an important role in the occurrence, development, and metastasis of tumors, as well as the prognosis of tumor malignancy (Du et al., 2008; Leung et al., 2010). In this study, we analyzed the relationship between CD44 expression and hepatoblastoma in children.

\section{MATERIAL AND METHODS}

\section{General information}

In total, 30 paraffin-block specimens of menstrual resected hepatoblastoma were collected from the Pediatric Surgery Department attached to the Beijing Military General Hospital (submitted between October 2005 and 2013). All samples were pathologically confirmed (19 male and 11 female); the samples were obtained from patients aged between 9 months and 6 years. The American children malignant liver staging system was used to determine the stage of tumor (Dong et al., 2005). Two of the 30 cases of hepatoblastoma were in stage I, and 10,11, and 7 cases were classified into stage II, III, and IV, respectively. Ten normal liver tissues were employed as the control samples (2 male and 8 female); the patients (who samples were obtained from) were aged between 6 months and 10 years (average age, 2.5 years). This study employed clinical laboratory studies. Informed consent was obtained by the patients themselves and their families. The study protocols were reviewed and approved by the ethics committee of the Beijing Military General Hospital.

\section{Main reagents and methods}

Mouse anti-human CD44 antibody was purchased from Abcam (Cambridge, UK).

\section{Experimental grouping}

Hepatoblastoma samples were divided into two groups based on the phase of disease; group A consisted of samples in the pathological stages I and II $(N=12)$, while group $B$ was comprised of samples in the pathological stages III and IV $(\mathrm{N}=18)$. Group $\mathrm{C}$ was composed of 10 normal liver tissue (control).

\section{Immunohistochemistry}

The paraffin block specimens were serially sectioned (thickness, $4 \mu \mathrm{m}$ ). The sections were subjected to streptomycin avidin - catalase connection (SP) immunohistochemistry. The sections 
were incubated with mouse anti-human CD44 monoclonal antibody (1:100), or phosphate buffered saline (PBS; negative control). The presence of brown granules in the cell membrane or cytoplasm indicated a positive expression of CD44; no less than $10 \%$ of the positive cells displayed CD44 expression (Cai et al., 2012).

\section{Statistical Methods}

The positive rate of CD44 expression was analyzed by the $x^{2}$ test. $P$ values $<0.05$ were considered to be statistically significant.

\section{RESULTS}

A positive CD44 expression in hepatoblastoma indicated staining of the cell membrane or cytoplasm (Figure 1). The rate of CD44-positive expression in groups A and B was $41.7 \%(5 / 12)$ and $83.3 \%(15 / 18)$, respectively; the CD44 expression was (statistically) significantly different between the two groups $\left(X^{2}=5.625 ; P<0.05\right)$. This indicated the correlation of CD44 expression with tumor staging (Table 1).

Level of CD44 expression in hepatoblastoma and normal liver tissues (Figure 2). The rate of CD44-positive cell expression in hepatoblastoma was $66.7 \%$ (20/30), while that in normal liver tissues was $20.0 \%$ (2/10); the CD44 expression differed statistically between the two groups $\left(X^{2}=\right.$ 4.848; $\mathrm{P}<0.05)$.

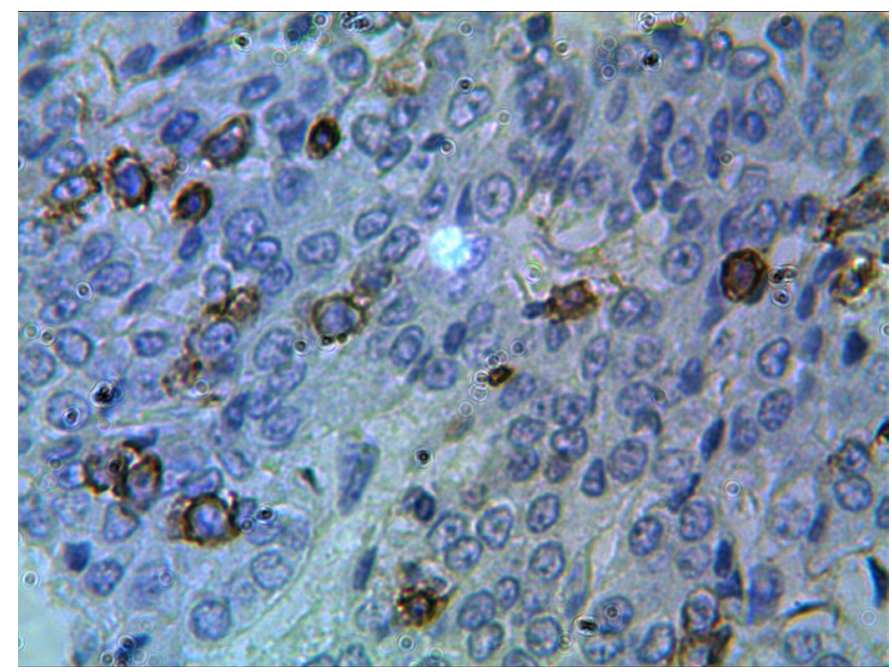

Figure 1. CD44-positive expression in hepatoblastoma SP 200X.

Table 1. The expression level of CD44 in hepatoblastoma and normal liver tissues.

\begin{tabular}{|c|c|c|c|c|}
\hline Group & CD44 positive (\%) & CD44 negative (\%) & $X^{2}$ value & $P$ value \\
\hline A & $5(41.7)$ & $7(58.3)$ & & \\
\hline B & $15(83.3)$ & $3(16.7)$ & 5.625 & $<0.05$ \\
\hline$A+B$ & $20(66.7)$ & $10(33.3)$ & & \\
\hline C & $2(20.0)$ & $8(80.0)$ & 4.848 & $<0.05$ \\
\hline
\end{tabular}




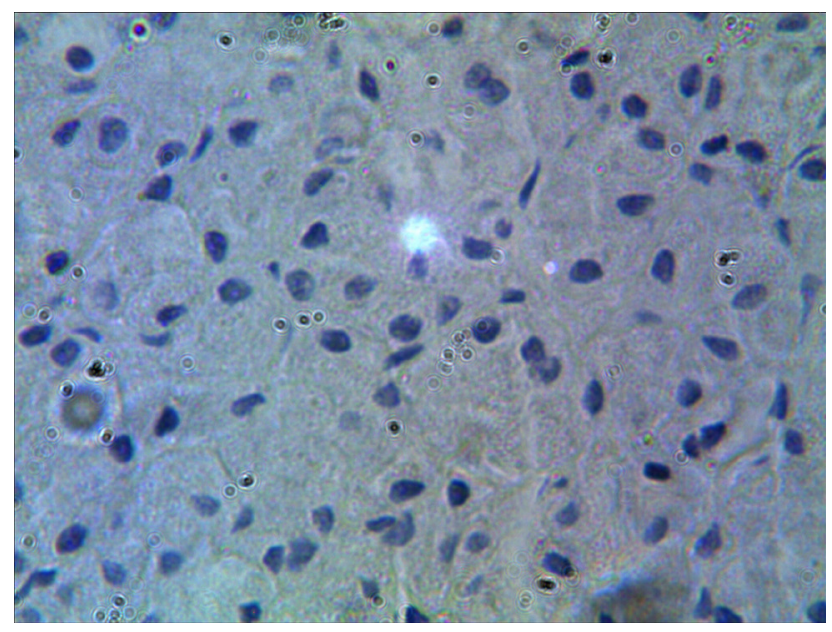

Figure 2. Negative expression of CD44 in normal liver tissues SP 200X.

\section{DISCUSSION}

Hepatoblastoma is one of the most common primary liver cancer affecting children, accounting for $50.0 \sim 70.0 \%$ of all primary liver tumors in children (Malogolowkin et al., 2011). The disease incidence from the embryonic stage to manifestation usually takes two years; therefore, the disease usually manifests around the age of 1 to 3 years. The major clinical significance of this disease is painless abdominal mass treatment, with a $90.0 \%$ increase in serum alpha-fetoprotein (AFP) levels, 10.0 to $20.0 \%$ cases displaying metastasis to the lung, hilar, brain, and bone, and a 2-year survival rate of $65.0 \%$ (Herzog et al., 2000). CD44 belongs to a family of cell adhesion molecules, with a wide variety of expression in the cells and tissue surface; this is encoded by a single class I transmembrane glycoprotein gene (Pasonen-Seppänen et al., 2012). CD44 forms a variety of isomers as a result of alternative splicing and post-translational modifications. CD44, in combination with a number of ligands on the cell surface, plays an important role in the physiological and pathological processes of a variety of cells. CD44 is highly expressed in many tumors, and is correlated to tumor growth, invasion, and metastasis, and homing of hematopoietic stem cells. It also serves as a marker for various tumor stem cells, acting as a potential target for cancer therapy. The biological function of CD44 expression and its regulatory patterns and growing conditions is different in different types of tumor (Ponta et al., 2003; Misra et al., 2013).

Previous studies have not reported the CD44 expression in HB liver tissues at home and abroad. We discovered a significantly higher expression of CD44 in HB liver tissues than in normal liver tissues $(P<0.05)$; the rate of positive CD44 expression was significantly lower in stage I and II tumors than that in stage III and IV tumors $(P<0.05)$. These results suggested that the CD44 expression was correlated with the occurrence, staging, and prognosis of $\mathrm{HB}$, which in turn played an important role in the growth and metastasis of tumor cells. This may be attributed to the involvement of CD44 in the binding of extracellular matrix and cells. A high expression of CD44positive tumor cells, combined with hyaluronic acid and other ECM ligands, imparts adhesion to the cells, which enhances the adhesion between tumor cells and the extracellular matrix (ECM). This increased the ease of access to growth factors from the ECM, thereby promoting the growth of tumor cells; when promoting tumor cells from the primary tumor, a low resistance path was formed 
for tumor cell metastasis. The tumor cells that reached the regional lymph nodes via the CD44 protein (and combined with the transparent hyaluronic acid) showed good growth. The degradation products produced at the time of combination also promoted tumor cell growth, and nourished the blood vessels. Therefore, CD44 was found to be significantly correlated with tumor growth and metastasis; this could be used as a specific indicator of lymphatic diffusion capacity and prognosis.

The development of molecular biology techniques could facilitate further studies into the regulation of CD44 and its related proteins in HB cells; this would provide a theoretical basis for early diagnosis and treatment of $\mathrm{HB}$, and open new avenues for its treatment.

\section{ACKNOWLEDGMENTS}

Research supported by the National Natural Science Foundation of China (\#81101860).

\section{Conflicts of interest}

The authors declare no conflict of interest.

\section{REFERENCES}

Cai HY, Yu B, Bai X, et al. (2012). Clinical significance of CD44 expression in colorectal cancer and metastatic lymph nodes. C. J. Med. Officers 40: 851-853. (Ref. não encontrada)

Du L, Wang H, He L, Zhang J, et al. (2008). CD44 is of functional importance for colorectal cancer stem cells. Clin. Cancer Res. 14: 6751-6760.

Dong Q, Xiao XM and Li Long (2005). Pediatric hepatobiliary surgery. People's Medical Publishing House, Beijing, 227-235. Helmberger TK, Ros PR, Mergo PJ, Tomczak R, et al. (1999). Pediatric liver neoplasms: a radiologic-pathologic correlation. Eur. Radiol. 9: 1339-1347.

Herzog CE, Andrassy RJ and Eftekhari F (2000). Childhood cancers: hepatoblastoma. Oncologist 5: 445-453.

Jang BI, Li Y, Graham DY and Cen P (2011). The role of CD44 in the pathogenesis, diagnosis, and therapy of gastric cancer. Gut Liver 5: 397-405.

Leung EL, Fiscus RR, Tung JW, Tin VP, et al. (2010). Non-small cell lung cancer cells expressing CD44 are enriched for stem cell-like properties. PLoS One 5: e14062.

Louderbough JM, Brown JA, Nagle RB and Schroeder JA (2011). CD44 promotes epithelial mammary gland development and exhibits altered localization during cancer progression. Genes Cancer 2: 771-781.

Malogolowkin MH, Katzenstein HM, Meyers RL, Krailo MD, et al. (2011). Complete surgical resection is curative for children with hepatoblastoma with pure fetal histology: a report from the Children's Oncology Group. J. Clin. Oncol. 29: 3301-3306.

Misra S, Ghatak S, Patil N, Dandawate PM, et al. (2013). Novel dual cyclooxygenase and lipoxygenase inhibitors targeting hyaluronan-CD44v6 pathway and inducing cytotoxicity in colon cancer cells. Bioorg. Med. Chem. 21: 2551-2559.

Pasonen-Seppänen S, Hyttinen JM, Rilla K, Jokela T, et al. (2012). Role of CD44 in the organization of keratinocyte pericellular hyaluronan. Histochem. Cell Biol. 137: 107-120.

Ponta H, Sherman L and Herrlich PA (2003). CD44: from adhesion molecules to signalling regulators. Nat. Rev. Mol. Cell Biol. 4: 33-45. 